Ultra-Wideband Short Pulse Electromagnetics 6, Edited by Mokole et al., Kluwer Academic/Plenum Publishers, 2003.

\title{
EFFECT OF THE INVERSION SYMMETRY OF DISPERSION RELATIONS ON FOUR-WAVE INTERACTIONS IN PHOTONIC CRYSTAL
}

\author{
Anatoli Babin and Alexander Figotin*
}

\section{INTRODUCTION}

The effect of the spatial periodicity on nonlinear optical processes has been a subject of intensive studies in physics literature ${ }^{1-27}$ and references therein. We study nonlinear wavepacket propagation assuming that its spatial range is much smaller than the dimension of the medium sample, therefore considering an infinite periodic medium. It is assumed that electromagnetic wave propagation is governed by the classical Maxwell equations

$$
\begin{gathered}
\nabla \times \mathbf{E}(\mathbf{r}, t)=-\frac{1}{c} \partial_{t} \mathbf{B}(\mathbf{r}, t)-\frac{4 \pi}{c} \mathbf{J}_{M}(\mathbf{r}, t), \nabla \cdot \mathbf{B}(\mathbf{r}, t)=0, \\
\nabla \times \mathbf{H}(\mathbf{r}, t)=\frac{1}{c} \partial_{t} \mathbf{D}(\mathbf{r}, t)+\frac{4 \pi}{c} \mathbf{J}_{E}(\mathbf{r}, t), \nabla \cdot \mathbf{D}(\mathbf{r}, t)=0,
\end{gathered}
$$

where $\mathbf{H}, \mathbf{E}, \mathbf{B}$, and $\mathbf{D}$ are respectively the magnetic and electric fields, magnetic and electric inductions; $\mathbf{J}_{E}$ and $\mathbf{J}_{M}$ are impressed electric and so called impressed magnetic currents; $\mathbf{r}=\left(x_{1}, x_{2}, x_{3}\right) \in \mathbf{R}^{3}$ is the position vector. It is also assumed that there are no free electric and magnetic charges, and, consequently, the fields $\mathbf{B}$ and $\mathbf{D}$ are divergence free as indicated in the equations (1) and (2). The vectors $\mathbf{H}, \mathbf{E}, \mathbf{B}$ and $\mathbf{D}$ are naturally 3 -component. We also consider two special cases: (i) the 2-dimensional case when the fields do not depend on $x_{3}$; (ii) the 1-dimensional case when the fields do not depend on $x_{2}, x_{3}$. Hence, we will consider three cases with the dimension $d=1,2,3$. For simplicity, we consider nonmagnetic media, i.e.

$$
\mathbf{B}(\mathbf{r}, t)=\mu \mathbf{H}(\mathbf{r}, t), \mu=1 .
$$

The material relations between $\mathbf{D}$ and $\mathbf{E}$ are assumed to be of the standard form ${ }^{28}$

$$
\mathbf{D}=\mathbf{E}+4 \pi \mathbf{P}(\mathbf{r}, t ; \mathbf{E}),
$$

*A. Babin and A. Figotin, Department of Mathematics, University of California at Irvine, CA 92697. 
where the polarization $\mathbf{P}$ is a sum of its linear and the nonlinear parts

$$
\mathbf{P}(\mathbf{r}, t ; \mathbf{E}(\cdot))=\mathbf{P}^{(1)}(\mathbf{r}, t ; \mathbf{E}(\cdot))+\mathbf{P}_{\mathrm{NL}}(\mathbf{r}, t ; \mathbf{E}(\cdot)),
$$

with the nonlinear part $\mathbf{P}_{\mathrm{NL}}(\mathbf{r}, t ; \mathbf{E}(\cdot))$ being a cubic nonlinearity.

Following Ref. 29, we choose $\mathbf{D}, \mathbf{B}$ as primary variables and rewrite the Maxwell equations (1), (2) in rescaled dimensionless variables as follows:

$$
\partial_{t} \mathbf{U}=-i \mathbf{M U}+\alpha \mathcal{F}_{\mathrm{NL}}(\mathbf{U})-\mathbf{J} ; \mathbf{U}(t)=0 \text { for } t \leq 0,
$$

where

$$
\mathbf{U}=\left[\begin{array}{l}
\mathbf{D} \\
\mathbf{B}
\end{array}\right], \mathbf{M U}=i\left[\begin{array}{c}
\nabla \times \mathbf{B} \\
-\nabla \times\left(\boldsymbol{\eta}^{(\mathbf{1})} \mathbf{D}\right)
\end{array}\right], \mathbf{J}=4 \pi\left[\begin{array}{c}
\mathbf{J}_{E} \\
\mathbf{J}_{M}
\end{array}\right]
$$

$\boldsymbol{\eta}^{(\mathbf{1})}=\boldsymbol{\eta}^{(\mathbf{1})}(\mathbf{r})=\varepsilon^{-1}(\mathbf{r})$ is the impermeability tensor, $\mathcal{F}_{\mathrm{NL}}(\mathbf{U})$ is a cubic nonlinearity. All the fields $\mathbf{D}, \mathbf{B}, \mathbf{J}_{E}$, and $\mathbf{J}_{M}$ are assumed to be divergence free. Both $\varepsilon(\mathbf{r})$ and $\mathbf{P}_{\mathrm{NL}}(\mathbf{r})$ are assumed to be periodic in $\mathbf{r}$ with the lattice of periods being a cubic lattice with the lattice constant 1 . The parameter $\alpha$ in (6) evidently determines the relative significance of the nonlinearity. We assume that $\alpha \ll 1$, that is we study weakly nonlinear phenomena. More exactly, we study nonlinear regimes satisfying the following assumptions: (i) the amplitude of the wave component due to the nonlinearity does not exceed the amplitude of its linear component and (ii) the wave spatial range is much smaller than the dimension of the medium sample. Under these constraints the nonlinear processes are naturally described in terms of the Bloch modes and corresponding dispersion relations of the underlying linear periodic medium. ${ }^{29}$

\subsection{Bloch Expansion}

Basic spectral properties of the differential operator $\mathbf{M}$ with periodic coefficients are covered by the Floquet-Bloch theory. ${ }^{30,31}$ Namely, all the eigenvalues and the eigenmodes of $\mathbf{M}$ are parametrized by two indices: zone (band) number $n=1,2, \ldots$, and the quasimomentum $\mathbf{k}$ from the so called Brillouin zone. The Brillouin zone in our case is the cube $[-\pi, \pi]^{d}$. We assume that the positive eigenvalues are naturally ordered

$$
0 \leq \omega_{1}(\mathbf{k}) \leq \omega_{2}(\mathbf{k}) \leq \cdots, \quad \mathbf{k} \text { in }[-\pi, \pi]^{d} .
$$

Notice that the operator $\mathbf{M}$ has a property that if $\omega$ is an eigenfrequency then $-\omega$ is an eigenfrequency as well. To take into account the negative eigenfrequency we introduce pairs

$$
\bar{n}=(\zeta, n) \text { where } \zeta= \pm 1, n=1,2, \ldots,
$$

and set

$$
\omega_{\bar{n}}(\mathbf{k})=\zeta \omega_{n}(\mathbf{k}), \text { for } \bar{n}=(\zeta, n) .
$$


The corresponding Bloch eigenmodes $\tilde{\mathbf{G}}_{\bar{n}}(\mathbf{r}, \mathbf{k})$ satisfy the following relations

$$
\mathbf{M} \tilde{\mathbf{G}}_{\bar{n}}(\mathbf{r}, \mathbf{k})=\omega_{\bar{n}}(\mathbf{k}) \tilde{\mathbf{G}}_{\bar{n}}(\mathbf{r}, \mathbf{k}), \tilde{\mathbf{G}}_{\bar{n}}(\mathbf{r}+\mathbf{m}, \mathbf{k})=e^{i \mathbf{k} \cdot \mathbf{m}} \tilde{\mathbf{G}}_{\bar{n}}(\mathbf{r}, \mathbf{k}), \mathbf{m} \text { in } \mathbb{Z}^{d},
$$

and they are divergence-free. For every fixed quasimomentum $\mathbf{k}$ the eigenfunctions $\tilde{\mathbf{G}}_{\bar{n}}(\mathbf{r}, \mathbf{k})$ with different $\bar{n}$ form an orthonormal basis. For any vector field $\mathbf{U}(\mathbf{r})$ we introduce its Bloch modal expansion

$$
\mathbf{U}(\mathbf{r})=\frac{1}{(2 \pi)^{d}} \sum_{\bar{n}} \int_{[-\pi, \pi]^{d}} \tilde{U}_{\bar{n}}(\mathbf{k}) \tilde{\mathbf{G}}_{\bar{n}}(\mathbf{r}, \mathbf{k}) d \mathbf{k},
$$

with $\tilde{U}_{\bar{n}}(\mathbf{k})$ being the Bloch amplitudes of $\mathbf{U}$.

\subsection{First Nonlinear Response and Interacting Quads}

If the excitation current $\mathbf{J}$ is chosen as an appropriate wavepacket, the solution $\mathbf{U}^{(0)}$ to the linear (with $\alpha=0$ ) Maxwell equations becomes a zero order approximation to the solution of the nonlinear Maxwell equation (6). ${ }^{29}$ It can be represented as

$$
\mathbf{U}^{(0)}(t)=\sum_{\bar{n}} \frac{1}{(2 \pi)^{d}} \int_{[-\pi, \pi]^{d}} \tilde{V}_{\bar{n}}^{(0)}(\mathbf{k}, \tau) \mathbf{G}_{\bar{n}}(\mathbf{r}, \mathbf{k}) e^{-i \omega_{\bar{n}}(\mathbf{k}) t} d \mathbf{k}, \quad \tau=\varrho t,
$$

where $\tau$ is the "slow time" with $\rho \ll 1$, and $\tilde{V}_{\bar{n}}^{(0)}(\mathbf{k}, \tau)$ are slowly varying in time modal amplitudes of the time harmonic carrier waves $\mathbf{G}_{\bar{n}}(\mathbf{r}, \mathbf{k}) e^{-i \omega_{\bar{n}}(\mathbf{k}) t}$. The parameter $\varrho \sim$ $\Delta \omega / \omega_{0}$ is the relative bandwidth of $\mathbf{J}$, where $\Delta \omega$ and $\omega_{0}$ are respectively the bandwidth and the carrier frequency of $\mathbf{J}$.

Let us consider the first order approximation to $\mathbf{U}(t)$, namely

$$
\mathbf{U}(t)=\mathbf{U}^{(0)}(t)+\alpha \mathbf{U}^{(1)}(t)+O\left(\alpha^{2}\right)
$$

with $\mathbf{U}^{(1)}(t)$ being the first nonlinear response of the medium. Its Bloch expansion has the form, ${ }^{29}$

$$
\begin{gathered}
\mathbf{U}^{(1)}(t)=\sum_{\bar{n}} \frac{1}{(2 \pi)^{d}} \int_{[-\pi, \pi]^{d}} \tilde{V}_{\bar{n}}^{(1)}(\mathbf{k}, \tau) \mathbf{G}_{\bar{n}}(\mathbf{r}, \mathbf{k}) e^{-i \omega_{\bar{n}}(\mathbf{k}) t} d \mathbf{k}, \quad \tau=\varrho t \\
\tilde{V}_{\bar{n}}^{(1)}(\mathbf{k}, \tau)=\frac{1}{\varrho} \sum_{\bar{n}^{\prime}, \bar{n}^{\prime \prime}} \int_{0}^{\tau} \int_{[-\pi, \pi]^{d}} \exp \left\{i \phi_{\vec{n}}\left(\mathbf{k}, \mathbf{k}^{\prime}, \mathbf{k}^{\prime \prime}\right) \frac{\tau_{1}}{\varrho}\right\} \\
\breve{Q}_{\vec{n}}\left(\mathbf{k}, \mathbf{k}^{\prime}, \mathbf{k}^{\prime \prime}\right) \tilde{V}_{\bar{n}^{\prime}}^{(0)}\left(\mathbf{k}^{\prime}, \tau_{1}\right) \tilde{V}_{\bar{n}^{\prime \prime}}^{(0)}\left(\mathbf{k}^{\prime \prime}, \tau_{1}\right) \tilde{V}_{\bar{n}^{\prime \prime \prime}}^{(0)}\left(\mathbf{k}-\mathbf{k}^{\prime}-\mathbf{k}^{\prime \prime}, \tau_{1}\right) d \mathbf{k}^{\prime} d \mathbf{k}^{\prime \prime} d \tau_{1} \\
\phi_{\vec{n}}\left(\mathbf{k}, \mathbf{k}^{\prime}, \mathbf{k}^{\prime \prime}\right)=\omega_{\bar{n}}(\mathbf{k})-\omega_{\bar{n}^{\prime}}\left(\mathbf{k}^{\prime}\right)-\omega_{\bar{n}^{\prime \prime}}\left(\mathbf{k}^{\prime \prime}\right)-\omega_{\bar{n}^{\prime \prime \prime}}\left(\mathbf{k}-\mathbf{k}^{\prime}-\mathbf{k}^{\prime \prime}\right)
\end{gathered}
$$


where $\breve{Q}_{\vec{n}}$ is a coefficient depending on the indices $\vec{n}=\left(\bar{n}, \bar{n}^{\prime}, \bar{n}^{\prime \prime}, \bar{n}^{\prime \prime \prime}\right)$ and the quasimomenta $\mathbf{k}, \mathbf{k}^{\prime}, \mathbf{k}^{\prime \prime}$, and $\phi_{\vec{n}}$ is a $2 \pi$-periodic with respect to $\mathbf{k}, \mathbf{k}^{\prime}, \mathbf{k}^{\prime \prime}$ phase function.

The asymptotic behavior of $\tilde{V}_{\bar{n}}^{(1)}(\mathbf{k}, \tau)$ in (16) as $\varrho \rightarrow 0$ is determined primarily by the phase $\phi_{\vec{n}}$. Its form (17) signifies the well known fact that the nonlinear interactions for a cubic nonlinearity occur through quads of modes $(\bar{n}, \mathbf{k}),\left(\bar{n}^{\prime}, \mathbf{k}^{\prime}\right),\left(\bar{n}^{\prime \prime}, \mathbf{k}^{\prime \prime}\right)$ and $\left(\bar{n}^{\prime \prime \prime}, \mathbf{k}^{\prime \prime \prime}\right)$ with the corresponding dispersion relations $\omega_{\bar{n}}(\mathbf{k}), \omega_{\bar{n}^{\prime}}\left(\mathbf{k}^{\prime}\right), \omega_{\bar{n}^{\prime \prime}}\left(\mathbf{k}^{\prime \prime}\right)$ and $\omega_{\bar{n}^{\prime \prime \prime}}\left(\mathbf{k}^{\prime \prime \prime}\right)$. The representation (16), (17) explicitly takes into account the phase matching condition

$$
\mathbf{k}^{\prime \prime \prime}=\mathbf{k}-\mathbf{k}^{\prime}-\mathbf{k}^{\prime \prime}
$$

for the interacting modes following from the medium periodicity.

To analyze the interactions, we look at the impact of a triad of modes $\left(\bar{n}^{\prime}, \mathbf{k}^{\prime}\right),\left(\bar{n}^{\prime \prime}, \mathbf{k}^{\prime \prime}\right)$ and $\left(\bar{n}^{\prime \prime \prime}, \mathbf{k}-\mathbf{k}^{\prime}-\mathbf{k}^{\prime \prime}\right)$ onto a mode $(\bar{n}, \mathbf{k})$, and observe that the amplitude of the first nonlinear response $\tilde{V}_{\bar{n}}^{(1)}(\mathbf{k}, \tau)$ depends on the amplitudes $\tilde{V}_{\bar{n}^{\prime}}^{(0)}\left(\mathbf{k}^{\prime}, \tau_{1}\right), \tilde{V}_{\bar{n}^{\prime \prime}}^{(0)}\left(\mathbf{k}^{\prime \prime}, \tau_{1}\right)$ and $\tilde{V}_{\bar{n}^{\prime \prime \prime}}^{(0)}\left(\mathbf{k}-\mathbf{k}^{\prime}-\mathbf{k}^{\prime \prime}, \tau_{1}\right), \tau_{1} \leq \tau$, of the linear response. Let us fix a $\tau>0$, and denote the contribution of amplitudes $\tilde{V}_{\bar{n}^{\prime}}^{(0)}\left(\mathbf{k}^{\prime}\right), \tilde{V}_{\bar{n}^{\prime \prime}}^{(0)}\left(\mathbf{k}^{\prime \prime}\right)$, and $\tilde{V}_{\bar{n}^{\prime \prime \prime}}^{(0)}\left(\mathbf{k}-\mathbf{k}^{\prime}-\mathbf{k}^{\prime \prime}\right)$ to the amplitude $\tilde{V}_{\bar{n}}^{(1)}(\mathbf{k})$ by $\tilde{V}_{\bar{n}}^{(1)}\left(\bar{n}, \bar{n}^{\prime}, \bar{n}^{\prime \prime}, \bar{n}^{\prime \prime \prime}, \mathbf{k}^{\prime \prime}, \mathbf{k}^{\prime}, \mathbf{k}\right)$. It is shown in Ref. 29 that for $\varrho \rightarrow 0$ the first nonlinear response $\tilde{V}_{\bar{n}}^{(1)}$ vanishes as a power of $\varrho$, namely

$$
\tilde{V}_{\bar{n}}^{(1)}\left(\bar{n}, \bar{n}^{\prime}, \bar{n}^{\prime \prime}, \bar{n}^{\prime \prime \prime}, \mathbf{k}^{\prime \prime}, \mathbf{k}^{\prime}, \mathbf{k}\right) \sim \varrho^{q}, \varrho \rightarrow 0 \text { where } 0<q \leq \infty,
$$

where the index $q$ describes the intensity of the interaction. If, for instance, the modes $(\bar{n}, \mathbf{k}),\left(\bar{n}^{\prime}, \mathbf{k}^{\prime}\right),\left(\bar{n}^{\prime \prime}, \mathbf{k}^{\prime \prime}\right)$, and $\left(\bar{n}^{\prime \prime \prime}, \mathbf{k}^{\prime \prime \prime}\right)$ are chosen "at random" then the above index $q$ is infinite, and consequently the mode interaction is weaker than any power of $\varrho$ as $\varrho \rightarrow$ 0 . But for special choices of quads the corresponding indices $q$ can be finite with more appreciable nonlinear interactions. When $q$ is finite, following Ref. 29 we introduce the index of interaction $q_{0}$ for the quad of modes by

$$
q_{0}=q_{0}\left(\vec{n}, \mathbf{k}^{\prime \prime}, \mathbf{k}^{\prime}, \mathbf{k}\right)=q+1, \quad \vec{n}=\left(\bar{n}, \bar{n}^{\prime}, \bar{n}^{\prime \prime}, \bar{n}^{\prime \prime \prime}\right) .
$$

It turns out that the index $q_{0}$ takes on only a few universal values, most important of which are collected in tables of Section 3. Note that a smaller interaction index $q_{0}$ corresponds to a stronger interaction.

The formula (16) suggests that the integral

$$
\begin{gathered}
I_{\vec{n}}(\mathbf{k}, \tau)=\frac{1}{\varrho} \int_{0}^{\tau} \int_{[-\pi, \pi]^{3}} \exp \left\{i \phi_{\vec{n}}\left(\mathbf{k}, \mathbf{k}^{\prime}, \mathbf{k}^{\prime \prime}\right) \frac{\tau_{1}}{\varrho}\right\} A\left(\mathbf{k}, \mathbf{k}^{\prime}, \mathbf{k}^{\prime \prime}, \tau_{1}\right) d \mathbf{k}^{\prime} d \mathbf{k}^{\prime \prime} d \tau_{1}, \\
A\left(\mathbf{k}, \mathbf{k}^{\prime}, \mathbf{k}^{\prime \prime}, \tau_{1}\right)=\tilde{Q}_{\vec{n}}(\mathbf{k}) \tilde{V}_{\bar{n}^{\prime}}^{(0)}\left(\mathbf{k}^{\prime}, \tau_{1}\right) \tilde{V}_{\bar{n}^{\prime \prime}}^{(0)}\left(\mathbf{k}^{\prime \prime}, \tau_{1}\right) \tilde{V}_{\bar{n}^{\prime \prime \prime}}^{(0)}\left(\mathbf{k}-\mathbf{k}^{\prime}-\mathbf{k}^{\prime \prime}, \tau_{1}\right)
\end{gathered}
$$

represents the nonlinear impact of the triad of modes $\left(\bar{n}^{\prime}, \mathbf{k}^{\prime}\right),\left(\bar{n}^{\prime \prime}, \mathbf{k}^{\prime \prime}\right),\left(\bar{n}^{\prime \prime \prime}, \mathbf{k}^{\prime \prime \prime}\right)$ onto the mode $(\bar{n}, \mathbf{k})$. We refer to integrals (21) as the oscillatory interaction integrals. Such 
interaction process is known in nonlinear optics as four wave mixing. We would like to emphasize that since the propagating wavepacket has a finite spatial range, its spectrum contains a continuum of modes all of which are involved in the four wave mixing. But for small $\varrho$ almost all interactions are insignificant except for a few. ${ }^{29}$

\subsection{Selection Rules}

To single out and classify significant nonlinear interactions one has to study the asymptotic behavior of the integrals (21) as $\varrho \rightarrow 0$. An analysis of the interaction integrals $I_{\vec{n}}(\mathbf{k}, \tau)$ of the form (21) carried out in Ref. 29 shows that a stronger nonlinear interaction can be found with the help of three selection rules. The first one, known as the phase matching condition (18), is already built in the very form of $I_{\vec{n}}(\mathbf{k}, \tau)$. The second selection rule, known as the frequency matching condition (FMC), is

$$
\phi_{\vec{n}}\left(\mathbf{k}, \mathbf{k}^{\prime}, \mathbf{k}^{\prime \prime}\right)=0
$$

or, in view of (17),

$$
\omega_{\bar{n}}(\mathbf{k})-\omega_{\bar{n}^{\prime}}\left(\mathbf{k}^{\prime}\right)-\omega_{\bar{n}^{\prime \prime}}\left(\mathbf{k}^{\prime \prime}\right)-\omega_{\bar{n}^{\prime \prime \prime}}\left(\mathbf{k}-\mathbf{k}^{\prime}-\mathbf{k}^{\prime \prime}\right)=0 .
$$

It is shown in Ref. 29 that if (24) is not fulfilled, the magnitude of the interaction integral $I_{\vec{n}}(\mathbf{k}, \tau)$ would be of order $\varrho^{q_{0}+1}$ compared with $\varrho^{q_{0}}$ if $(24)$ is satisfied. Therefore, the condition (24) must hold for stronger interactions.

The third selection rule, called the group velocity matching condition, ${ }^{29}$ follows from the asymptotic analysis of the integral

$$
I_{\vec{n}}(\mathbf{k})=\frac{1}{\varrho} \int_{[-\pi, \pi]^{d}} \exp \left\{i \phi_{\vec{n}}\left(\mathbf{k}, \mathbf{k}^{\prime}, \mathbf{k}^{\prime \prime}\right) \frac{\tau_{1}}{\varrho}\right\} A\left(\mathbf{k}, \mathbf{k}^{\prime}, \mathbf{k}^{\prime \prime}, \tau_{1}\right) d \mathbf{k}^{\prime} d \mathbf{k}^{\prime \prime},
$$

which is an interior integral in (21). Evidently, the integral involves the large parameter $\frac{1}{\varrho}$ for $\varrho \ll 1$, and one can apply the stationary phase method. According to the method, for any fixed $\mathbf{k}$ the asymptotic behavior of $I_{\vec{n}}(\mathbf{k})$ as $\varrho \rightarrow 0$ is determined by critical points of the phase function $\phi_{\vec{n}}\left(\mathbf{k}, \mathbf{k}^{\prime}, \mathbf{k}^{\prime \prime}\right)$, i.e. the points $\left(\mathbf{k}_{*}^{\prime}, \mathbf{k}_{*}^{\prime \prime}\right)$ solving the following two $d$-component equations

$$
\nabla_{k^{\prime}} \phi_{\vec{n}}\left(\mathbf{k}, \mathbf{k}^{\prime}, \mathbf{k}^{\prime \prime}\right)=0, \nabla_{k^{\prime \prime}} \phi_{\vec{n}}\left(\mathbf{k}, \mathbf{k}^{\prime}, \mathbf{k}^{\prime \prime}\right)=0
$$

In view of (17), the equations (26) can be recast as

$$
\nabla \omega_{\bar{n}^{\prime}}\left(\mathbf{k}_{*}^{\prime}\right)=\nabla \omega_{\bar{n}^{\prime \prime}}\left(\mathbf{k}_{*}^{\prime \prime}\right)=\nabla \omega_{\bar{n}^{\prime \prime \prime}}\left(\mathbf{k}_{*}^{\prime \prime \prime}\right), \mathbf{k}_{*}^{\prime \prime \prime}=\mathbf{k}-\mathbf{k}_{*}^{\prime}-\mathbf{k}_{*}^{\prime \prime},
$$

justifying the name group velocity matching condition $(G V M)$ since $\nabla \omega_{\bar{n}^{\prime}}(\mathbf{k})$ is the group velocity of the $\mathbf{k}$-component of the linear wavepacket. 


\section{OUTLINE OF MAIN RESULTS}

The stationary phase method analysis shows that the contribution of a critical point $\left(\mathbf{k}_{*}^{\prime}, \mathbf{k}_{*}^{\prime \prime}\right)$ to the integral $I_{\vec{n}}(\mathbf{k})$ is of order $\varrho^{q_{0}-1}$ where $q_{0}$ is determined by the properties of the phase function $\phi_{\vec{n}}$ at this point. ${ }^{29}$ Notice that the critical point $\left(\mathbf{k}_{*}^{\prime}, \mathbf{k}_{*}^{\prime \prime}\right)$ does not depend on the amplitude $A\left(\mathbf{k}^{\prime}, \mathbf{k}^{\prime \prime}\right)$ provided it is smooth, but the magnitude of the interaction clearly is proportional to $A\left(\mathbf{k}_{*}^{\prime}, \mathbf{k}_{*}^{\prime \prime}\right)$. Such points must satisfy (27) whereas all other points and corresponding interactions are negligible. Observe that the vector equation (27) yields $2 d$ scalar equations for $2 d$ variables $\mathbf{k}^{\prime}, \mathbf{k}^{\prime \prime}$ and may have several solutions. The FMC condition (24) imposes one more constraint on $\mathbf{k}_{*}^{\prime}, \mathbf{k}_{*}^{\prime \prime}$.

Note that for a fixed $\mathbf{k}$ the conditions (27) together with (24) impose $2 d+1$ equations on $2 d$ variables, and in a generic case one cannot expect these conditions to be satisfied. Of course, there are cases when $2 d+1$ equations on $2 d$ variables have a solution, but such cases are not typical. It turns out, though, that under the inversion (spectral) symmetry condition

$$
\omega_{n}(\mathbf{k})=\omega_{n}(-\mathbf{k}), \mathbf{k} \in[-\pi, \pi]^{d}, n=1,2, \ldots,
$$

the equations have solutions. The inversion symmetry condition (28) on the dispersion relations is a special case of Wigner time-reversal symmetry ${ }^{32}$ ), and it always holds, for instance, for any nonmagnetic periodic medium. Note that (28) implies

$$
\nabla \omega_{n}(\mathbf{k})=-\nabla \omega_{n}(-\mathbf{k}) .
$$

In this paper we study the case when (28) holds and show that the overdetermined system (27), (23) always has a solution. In particular, for a given $(\bar{n}, \mathbf{k})$ the quad of strongly interacting modes solving the overdetermined system (27), (23) satisfies the following relations

$$
n^{\prime}=n^{\prime \prime}=n^{\prime \prime \prime}=n \text {. }
$$

In other words, the quad of modes belongs to the same band. In addition to that, the quasimomenta and the signs $\zeta$ in the index $\bar{n}=(\zeta, n)$ of $\omega_{\bar{n}}(\mathbf{k})$ are chosen as follows:

$$
\mathbf{k}_{*}^{\prime \prime \prime}=-\mathbf{k}, \mathbf{k}_{*}^{\prime}=\mathbf{k}_{*}^{\prime \prime}=\mathbf{k}, \quad \zeta^{\prime \prime \prime}=-\zeta, \zeta^{\prime}=\zeta^{\prime \prime}=\zeta .
$$

If $\zeta$ are chosen as in (31) the phase function (17) takes the form

$$
\phi_{\vec{n}}\left(\mathbf{k}, \mathbf{k}^{\prime}, \mathbf{k}^{\prime \prime}\right)= \pm\left[-\omega_{n}(\mathbf{k})+\omega_{n^{\prime}}\left(\mathbf{k}^{\prime}\right)+\omega_{n^{\prime \prime}}\left(\mathbf{k}^{\prime \prime}\right)-\omega_{n^{\prime \prime \prime}}\left(\mathbf{k}-\mathbf{k}^{\prime}-\mathbf{k}^{\prime \prime}\right)\right],
$$

and it is easy to examine that (27) and (24) are satisfied. There are two more quads similar to (31) with the same property, namely

$$
\mathbf{k}_{*}^{\prime}=-\mathbf{k}, \mathbf{k}_{*}^{\prime \prime}=\mathbf{k}_{*}^{\prime \prime \prime}=\mathbf{k}, \zeta^{\prime \prime}=\zeta^{\prime \prime \prime}=\zeta, \zeta^{\prime}=-\zeta,
$$

and

$$
\mathbf{k}_{*}^{\prime \prime}=-\mathbf{k}, \mathbf{k}_{*}^{\prime}=\mathbf{k}_{*}^{\prime \prime \prime}=\mathbf{k}, \zeta^{\prime \prime \prime}=\zeta^{\prime}=\zeta, \zeta^{\prime \prime}=-\zeta .
$$


In the nonlinear optics this type of interactions is known to occur in the process of a degenerate four-wave mixing, Ref. 28 p. 232.

Let us gauge now the intensity of nonlinear interactions using the interaction index $q_{0}$ and find the corresponding quads of modes. For simplicity, we consider $d=1$ and $d=2$. If we allow $\mathbf{k}$ in the system (24), (26) to be a variable together with $\mathbf{k}^{\prime}, \mathbf{k}^{\prime \prime}$ and if the dispersion relations do not have the inversion symmetry (28) and are generic, the set of all solutions is a $d-1$ dimensional manifold in the $\mathbf{k}, \mathbf{k}^{\prime}, \mathbf{k}^{\prime \prime}$-space. Let us denote this manifold by $\Xi=\Xi(\vec{n})$. Every point $\left(\mathbf{k}, \mathbf{k}^{\prime}, \mathbf{k}^{\prime \prime}\right)$ in $\Xi(\vec{n})$ corresponds to a strongly interacting quad.

Remarkably, in the presence of the inversion symmetry (28), if $n=n^{\prime}=n^{\prime \prime}=$ $n^{\prime \prime \prime}$ and the signs $\zeta$ are as in (31), (32) or (33), the manifold $\Xi(\vec{n})$ is $d$-dimensional. In addition to that, the index $q_{0}\left(\vec{n}, \mathbf{k}, \mathbf{k}^{\prime}, \mathbf{k}^{\prime \prime}\right)$ of a strongly interacting quad depends on the rank of the Hessian $\nabla^{2} \phi_{\vec{n}}\left(\mathbf{k}, \mathbf{k}^{\prime}, \mathbf{k}^{\prime \prime}\right)$ of the phase function at the point. For a typical quad from $\Xi(\vec{n})$ the Hessian at the point is not degenerate, its rank equals $2 d$, and the interaction index $q_{0}=d$. Let us denote by $\Xi^{\prime}=\Xi^{\prime}(\vec{n})$ a submanifold of $\Xi(\vec{n})$ on which the determinant of the Hessian of $\phi_{\vec{n}}\left(\mathbf{k}, \mathbf{k}^{\prime}, \mathbf{k}^{\prime \prime}\right)$ with respect to $\mathbf{k}^{\prime}, \mathbf{k}^{\prime \prime}$ vanishes, and by $\Xi^{\prime \prime}=\Xi^{\prime \prime}(\vec{n})$ the submanifold of $\Xi^{\prime}(\vec{n})$ on which the rank of the Hessian is $2 d-2$. As a rule, interactions corresponding to $\Xi^{\prime \prime}$ are stronger than the ones corresponding to $\Xi^{\prime}$, and interactions corresponding to $\Xi^{\prime}$ are stronger than the ones corresponding to $\Xi$. Our analysis shows that if the inversion symmetry (28) holds and $n=n^{\prime}=n^{\prime \prime}=n^{\prime \prime \prime}$ then the manifold $\Xi^{\prime \prime}(\vec{n})$ is always non-empty and its dimension is $d-1$. For quads from $\Xi^{\prime \prime}$ generically the index $q_{0}=d-\frac{1}{3}$, for $d=1,2$. When the inversion symmetry condition (28) does not hold, $\Xi^{\prime \prime}(\vec{n})$ may be empty. In fact, it is always empty for a generic onedimensional medium, $d=1$. For a generic two-dimensional medium, $d=2$, without inversion symmetry it may be non-empty only for the quads related to the Third Harmonic Generation when

$$
n^{\prime}=n^{\prime \prime}=n^{\prime \prime \prime}, \mathbf{k}_{*}^{\prime}=\mathbf{k}_{*}^{\prime \prime}=\mathbf{k}_{*}^{\prime \prime \prime}=\frac{1}{3} \mathbf{k}, \zeta=\zeta^{\prime}=\zeta^{\prime \prime}=\zeta^{\prime \prime \prime} .
$$

In addition, if $\Xi^{\prime \prime}$ is non-empty, it may consist of only several points, that is the dimension of $\Xi^{\prime \prime}$ is zero, and the interaction index $q_{0}=\frac{5}{3}$.

Therefore, the symmetry condition (28) dramatically changes the structure and the dimension of the manifold $\Xi(\vec{n})$ of solutions $\left(\mathbf{k}, \mathbf{k}^{\prime}, \mathbf{k}^{\prime \prime}\right)$ to the selections rules (23), (24) describing strongly interacting modes. Namely, for any $(\bar{n}, \mathbf{k})$ there always exist a quad of modes involving $(\bar{n}, \mathbf{k})$ and satisfying all the selection rules. In addition to that, the interactions are stronger than in the absence of the inversion symmetry (see tables in Section 4).

Recall that the optical Kerr effect (Ref. 28 p. 26) involves a quadruple of interacting modes with frequencies $-\omega_{S}, \omega_{P},-\omega_{P}, \omega_{S}$. Similar interactions occur for a periodic medium with the inversion symmetry $(28)$ for quads satisfying all the selection rules. Indeed, let us set

$$
\omega_{S}=\omega_{n}(\mathbf{k})=\omega_{n^{\prime}}\left(\mathbf{k}^{\prime}\right), \omega_{P}=\omega_{n^{\prime}}\left(\mathbf{k}^{\prime \prime}\right)=\omega_{n^{\prime \prime \prime}}\left(\mathbf{k}-\mathbf{k}^{\prime}-\mathbf{k}^{\prime \prime}\right)
$$




$$
n^{\prime \prime \prime}=n^{\prime \prime}, n=n^{\prime}, \mathbf{k}=\mathbf{k}^{\prime}, \mathbf{k}^{\prime \prime \prime}=-\mathbf{k}^{\prime \prime}, \zeta=\zeta^{\prime}=\zeta^{\prime \prime}=-\zeta^{\prime \prime \prime} .
$$

Then the phase function takes the form

$$
\left.\phi_{\vec{n}}\left(, \mathbf{k}, \mathbf{k}^{\prime}, \mathbf{k}^{\prime \prime}\right)\right)=-\omega_{n}(\mathbf{k})+\omega_{n^{\prime}}\left(\mathbf{k}^{\prime}\right)+\omega_{n^{\prime \prime}}\left(\mathbf{k}^{\prime \prime}\right)-\omega_{n^{\prime \prime \prime}}\left(\mathbf{k}-\mathbf{k}^{\prime}-\mathbf{k}^{\prime \prime}\right) .
$$

This function always satisfies FMC; obviously GVM holds for $\mathbf{k}^{\prime \prime}=\mathbf{k}^{\prime}, n^{\prime \prime}=n^{\prime}$, but GVM may also hold for the case $n^{\prime \prime} \neq n^{\prime}, \mathbf{k}^{\prime \prime} \neq \mathbf{k}^{\prime}=\mathbf{k}$ if the equation $\nabla \omega_{n^{\prime}}\left(\mathbf{k}^{\prime}\right)=\nabla \omega_{n^{\prime \prime}}\left(\mathbf{k}^{\prime \prime}\right)$ is satisfied. The last condition yields $d$ scalar equations on $d$ variables $\mathbf{k}^{\prime \prime}$ and may be satisfied for several $\mathbf{k}^{\prime \prime}=\mathbf{k}^{\prime \prime}(\mathbf{k})$.

\section{OSCILLATORY INTEGRALS}

For $\varrho \rightarrow 0$ the interaction integral of equation (25) involves a rapidly oscillating function $\exp \left\{i \phi_{\vec{n}}\left(\mathbf{k}, \mathbf{k}^{\prime}, \mathbf{k}^{\prime \prime}\right) \frac{\tau 1}{\varrho}\right\}$. To analyze its asymptotic behavior we consider the integral

$$
I(\varrho, \mathbf{k})=\int_{\mathbf{R}^{d}} e^{i \Phi(\mathbf{k}, \mathbf{s}) / \varrho} A(\mathbf{k}, \mathbf{s}) d \mathbf{s}, \mathbf{s}=\left(\mathbf{k}^{\prime}, \mathbf{k}^{\prime \prime}\right), \varrho \rightarrow 0,
$$

where the the integration variable $\mathbf{s}$ has dimension $d_{I}=2 d, \mathbf{k}$ is a continuous parameter, and the phase function is

$$
\Phi(\mathbf{k}, \mathbf{s})=\phi_{\vec{n}}\left(\mathbf{k}, \mathbf{k}^{\prime}, \mathbf{k}^{\prime \prime}\right)
$$

with $\phi_{\vec{n}}$ being defined by (17). The phase $\Phi(\mathbf{k}, \mathbf{s})$ depends on a $d$-dimensional parameter $\mathbf{k}$ and is assumed to satisfy the FMC equation $\Phi(\mathbf{k}, \mathbf{s})=0$ that imposes one constraint on $d$ parameters. Hence, generally speaking, $\Phi(\mathbf{k}, \mathbf{s})$ is a $d-1$ parametric family of phase functions. Note that the integral (25) has the factor $\varrho^{-1}$ which is not included in the integral (36).

Let us review briefly the main points of the stationary phase method for oscillatory integrals of the form

$$
I(\varrho)=\int_{\mathbf{R}^{d_{I}}} e^{i \Phi(\mathbf{s}) / \varrho} A(\mathbf{s}) d \mathbf{s}, \varrho \rightarrow 0,
$$

where $A(\mathbf{s})$ is assumed to be an infinitely smooth with a finite support. According to the stationary phase method, the main contribution to $I(\varrho)$ for $\varrho \rightarrow 0$ comes from small neighborhoods of critical points of the phase $\Phi(\mathbf{s})$, that is points $\mathbf{s}_{*}$ satisfying the equation

$$
\nabla_{\mathbf{s}} \Phi(\mathbf{s})=0
$$

and those contributions are proportional to powers of $\varrho$. The corresponding coefficients before the powers are written in terms of values of $\Phi(\mathbf{s}), A(\mathbf{s})$ and its derivatives at critical points $\mathbf{s}_{*}$. Since (39) is a system of $d_{I}$ equations for $d_{I}$ variables, for a generic $\Phi(\mathbf{s})$ there is a finite number of such points. 


\subsection{Standard Asymptotic Approximations}

In this subsection we list some standard asymptotic formulas used in our analysis. One of the most important characteristics of a critical point $\mathbf{s}_{*}$ is the rank of the Hessian $\Phi^{\prime \prime}\left(\mathbf{s}_{*}\right)$. The formulas provided below are for the cases when the rank of the Hessian is $d_{I}$ or $d_{I}-1$.

\subsubsection{Non-Degenerate Points}

When the rank of the Hessian is $d_{I}$ we have a non-degenerate critical point $\mathbf{s}_{*}$, or a critical point of type $A_{1}$. All the eigenvalues $\mu_{1}, \ldots, \mu_{d_{I}}$ of the Hessian at this point are non-zero, and the following classical formula holds

$$
I(\varrho)=b_{0} \varrho^{\frac{d_{I}}{2}} b_{\mathcal{T}} A\left(\mathbf{s}_{*}\right) e^{i \Phi\left(\mathbf{s}_{*}\right) / \varrho}+O\left(\varrho^{\frac{d_{I}}{2}+1}\right), \varrho \rightarrow 0,
$$

with

$$
b_{0}=\frac{(2 \pi)^{\frac{d_{I}}{2}}}{\sqrt{\left|\operatorname{det} \Phi^{\prime \prime}\left(\mathbf{s}_{*}\right)\right|}} \exp \left\{i \eta \Phi\left(\mathbf{s}_{*}\right)+\frac{i \pi}{4} \operatorname{sign}\left[\Phi^{\prime \prime}\left(\mathbf{s}_{*}\right)\right]\right\},
$$

where $\operatorname{sign}\left[\Phi^{\prime \prime}\left(\mathbf{s}_{*}\right)\right]$ is the signature of the matrix $H$ (the algebraic sum of the signs of the matrix eigenvalues).

\subsubsection{Simplest degenerate points}

When the rank of the Hessian is $d_{I}-1$ we have a critical point $\mathbf{s}_{*}$ of the type $A_{p}$, $p>1$. One of the eigenvalues of the Hessian vanishes, i.e. $\mu_{d_{I}}=0$. In this case along a curve tangent to the null-space of $\Phi^{\prime \prime}\left(\mathbf{s}_{*}\right)$ the function $\Phi(\mathbf{s})$ vanishes at $\mathbf{s}_{*}$ as $\left|\mathbf{s}-\mathbf{s}_{*}\right|^{p+1}$ with $p>1$. For a critical point of the class $\mathcal{T}=A_{p}$ the leading term of the asymptotics takes the form (see, for example, Ref. 33, Section 6.1):

$$
I(\varrho)=\varrho^{\frac{d_{I}-1}{2}+\frac{1}{p+1}} b_{\mathcal{T}} A\left(\mathbf{s}_{*}\right) e^{i \eta \Phi\left(\mathbf{s}_{*}\right)}+O\left(\varrho^{\frac{d_{I}-1}{2}+\frac{2}{p+1}}\right)
$$

where the coefficient $b_{\mathcal{T}}=b_{A_{p}}$ is determined by the phase $\Phi$ at $\mathbf{s}_{*}$ by a formula similar to (41) and the oscillatory index is

$$
q_{\mathcal{T}}=q_{A_{p}}=\frac{d_{I}-1}{2}+\frac{1}{p+1} .
$$

Formula (43) for the oscillatory index holds as well for a non-degenerate point with $p=1$.

When the rank of the Hessian is $d_{I}-2$ a more involved analysis of critical points $\mathbf{s}_{*}$ is needed. For the asymptotic expansions of oscillatory integrals with a general phase functions see Refs. 34, 35, and references therein. Our analysis shows that to find the leading terms of asymptotics in generic situations when $\mathbf{k}$ is one-, two-, or three-dimensional variable it is sufficient to consider relatively simple singularities and the values of the index $q_{\mathcal{T}}$ can be found in these cases. 


\subsection{Oscillatory Integrals With Parameters}

In this subsection we focus on the oscillatory integrals (36) that depend on the parameter $\mathbf{k}$. To make the results more transparent, we consider first a general phase $\Phi(\mathbf{k}, \mathbf{s})$ and do not assume the special structure (37). Then we consider the case of (37) not assuming the inversion symmetry (28), and, finally, we will consider the case when (28) holds.

\subsubsection{General Phase Function}

Let us consider the integral (36) with a general phase function $\Phi(\mathbf{k}, \mathbf{s})$ that does not have the special structure of (17). This example serves as a basis for a comparison and indicates the effect coming from the structure of (17).

Case 1. Let $d_{I}=2 d=2$. In this case the GVM conditions (26) and the FMC condition (23) yield 3 equations on three one-dimensional parameters $\mathbf{k}, \mathbf{k}^{\prime}, \mathbf{k}^{\prime \prime}$ selecting several values $\left(\mathbf{k}_{*}, \mathbf{s}_{*}\right)$ of the variables $\mathbf{s}$ and $\mathbf{k}$. Hence, the manifold $\Xi$ has zero dimension, and for a fixed typical $\mathbf{k} \neq \mathbf{k}_{*}$ the system does not have a solution. In a generic case, the Hessian $\Phi^{\prime \prime}\left(\mathbf{k}_{*}, \mathbf{S}_{*}\right)$ with respect to $\mathbf{s}$ at these points is non-degenerate, $\operatorname{det} \Phi^{\prime \prime}\left(\mathbf{k}_{*}, \mathbf{s}_{*}\right) \neq 0$, therefore $\Xi^{\prime}$ is empty. The principal term of asymptotics of (36) by the classical stationary phase method (41) is of order $\varrho$.

Case 2. Let us take $d_{I}=4$. The vector equations (26) and (23) yield 5 scalar equations on three two-dimensional parameters $\mathbf{k}, \mathbf{k}^{\prime}, \mathbf{k}^{\prime \prime}$, that is 5 equations on 6 variables. Their solutions $\overrightarrow{\mathbf{k}}=\left(\mathbf{k}, \mathbf{k}^{\prime}, \mathbf{k}^{\prime \prime}\right)$ form a manifold $\Xi$ of the strongly interacting modes. In a generic case, $\Xi$ is a one-dimensional manifold (a curve). This manifold may have singular points (self-intersections). When $\mathbf{k}$ from this curve is fixed, we have several critical points $\mathbf{s}_{*}=$ $\left(\mathbf{k}_{*}^{\prime}, \mathbf{k}_{*}^{\prime \prime}\right)$. When the Hessian $\Phi^{\prime \prime}(\mathbf{k}, \mathbf{s})=\nabla_{k^{\prime} k^{\prime \prime}}^{2} \Phi\left(\mathbf{k}, \mathbf{k}^{\prime}, \mathbf{k}^{\prime \prime}\right)$ of $\Phi\left(\mathbf{k}, \mathbf{k}^{\prime}, \mathbf{k}^{\prime \prime}\right)$ with respect to $\mathbf{s}=\left(\mathbf{k}^{\prime}, \mathbf{k}^{\prime \prime}\right)$ is non-degenerate, i.e.

$$
\operatorname{det} \nabla_{k^{\prime} k^{\prime \prime}}^{2} \Phi\left(\mathbf{k}, \mathbf{k}_{*}^{\prime}, \mathbf{k}_{*}^{\prime \prime}\right) \neq 0,
$$

we have non-degenerate critical points $\mathbf{s}_{*}=\left(\mathbf{k}_{*}^{\prime}, \mathbf{k}_{*}^{\prime \prime}\right)$ of the phase function. According to (41) the contribution of such a point to the integral (36) is of order $\varrho^{2}$.

Since the interaction manifold $\Xi$ is one-dimensional, there can be points where

$$
\operatorname{det} \nabla_{k^{\prime} k^{\prime \prime}}^{2} \Phi\left(\mathbf{k}, \mathbf{k}_{*}^{\prime}, \mathbf{k}_{*}^{\prime \prime}\right)=0 .
$$

The solutions to (44) form the manifold $\Xi^{\prime}$. If $\operatorname{det} \nabla_{k^{\prime} k^{\prime \prime}}^{2} \Phi\left(\mathbf{k}, \mathbf{k}^{\prime}, \mathbf{k}^{\prime \prime}\right)$ changes the sign along the curve at such a point (this is a generic case), such a point cannot be eliminated by small perturbations of the dispersion function $\Phi(\mathbf{k}, \mathbf{s})$. Hence, the degeneration is robust and $\Xi^{\prime}$ may be non-empty. In a generic case, the rank of the Hessian is $d_{I}-1$, and a generic $\Phi(\mathbf{k}, \mathbf{s})$ at $\mathbf{s}=\mathbf{s}^{*}$ vanishes in the direction of the null-space of the Hessian at cubic rate, resulting in a singularity of the type $A_{2}$. According to (42) the contribution of such point is of order $\varrho^{11 / 6}$. 


\subsubsection{Phase Function of Four-Wave Interactions}

We consider now (36) with a phase function $\Phi(\mathbf{k}, \mathbf{s})$ that has the special structure given by (17). Since $\omega_{\bar{n}}(\mathbf{k})= \pm \omega_{n}(\mathbf{k})=\zeta \omega_{n}(\mathbf{k})$, where the sign is determined by the $\zeta$-component of $\bar{n}=(\zeta, n)$, we have by $(17)$

$$
\begin{aligned}
\Phi(\mathbf{k}, \mathbf{s}) & =\phi_{\vec{n}}\left(\mathbf{k}, \mathbf{k}^{\prime}, \mathbf{k}^{\prime \prime}\right) \\
& =\zeta \omega_{n}(\mathbf{k})-\zeta^{\prime} \omega_{n^{\prime}}\left(\mathbf{k}^{\prime}\right)-\zeta^{\prime \prime} \omega_{n^{\prime \prime}}\left(\mathbf{k}^{\prime \prime}\right)-\zeta^{\prime \prime \prime} \omega_{n^{\prime \prime \prime}}\left(\mathbf{k}-\mathbf{k}^{\prime}-\mathbf{k}^{\prime \prime}\right), \mathbf{s}=\left(\mathbf{k}^{\prime}, \mathbf{k}^{\prime \prime}\right) .
\end{aligned}
$$

If $n^{\prime \prime \prime} \neq n^{\prime \prime}$ or $n^{\prime \prime \prime} \neq n^{\prime}$, the analysis of (36) is similar to the analysis for a general phase function and it yields the same conclusions. If $d=1, d_{I}=2$, for a generic $\omega_{n}(\mathbf{k})$ even in the case $n^{\prime \prime \prime}=n^{\prime \prime}=n^{\prime}$ the situation is similar to the considered case of a general $\Phi(\mathbf{k}, \mathbf{s})$. Namely, in the case $d=1$ for a generic $\omega_{n}(\mathbf{k})$ there are only non-degenerate points, $\Xi^{\prime}$ is empty and the oscillatory integral (36) has the leading asymptotic term of order $\varrho^{1}$.

In the case $d=2$, for a generic $\omega_{n}(\mathbf{k})$ the manifold $\Xi$ is one-dimensional (if nonempty) and $\Xi^{\prime}$ is zero-dimensional (if non-empty). For a generic $\omega_{n}(\mathbf{k})$ points from $\Xi^{\prime}$ are of the type $A_{2}$, and we have a contribution from such a point of order $\varrho^{11 / 6}$ as for a general phase function. The special structure of the function $\Phi(\mathbf{k}, \mathbf{s})$ implies certain restrictions on the Hessian. The Hessian $\Phi^{\prime \prime}(\mathbf{k}, \mathbf{s})$ with respect to $\left(\mathbf{k}^{\prime}, \mathbf{k}^{\prime \prime}\right)=\mathbf{s}$ takes the block form

$$
\Phi^{\prime \prime}\left(\mathbf{k}^{\prime}, \mathbf{k}^{\prime \prime}\right)=-\left(\begin{array}{cc}
\zeta^{\prime} \omega_{n^{\prime}}^{\prime \prime}\left(\mathbf{k}^{\prime}\right)+\zeta^{\prime \prime \prime} \omega_{n^{\prime \prime \prime}}^{\prime \prime}\left(\mathbf{k}^{\prime \prime \prime}\right) & \zeta^{\prime \prime \prime} \omega_{n^{\prime \prime \prime}}^{\prime \prime}\left(\mathbf{k}^{\prime \prime \prime}\right) \\
\zeta^{\prime \prime \prime} \omega_{n^{\prime \prime \prime}}^{\prime \prime}\left(\mathbf{k}^{\prime \prime \prime}\right) & \zeta^{\prime \prime} \omega_{n^{\prime \prime}}^{\prime \prime}\left(\mathbf{k}^{\prime \prime}\right)+\zeta^{\prime \prime \prime} \omega_{n^{\prime \prime \prime}}^{\prime \prime \prime}\left(\mathbf{k}^{\prime \prime \prime}\right)
\end{array}\right)
$$

where $\mathbf{k}^{\prime \prime \prime}=\mathbf{k}-\mathbf{k}^{\prime}-\mathbf{k}^{\prime \prime}$

The case $n^{\prime \prime \prime}=n^{\prime \prime}=n^{\prime}$ is special. In this case when $\zeta^{\prime}=\zeta^{\prime \prime}=\zeta^{\prime \prime \prime}$ the GVM condition (27) has a special family of solutions

$$
\mathbf{k}_{*}^{\prime}=\mathbf{k}_{*}^{\prime \prime}=\mathbf{k}_{*}^{\prime \prime \prime}=\frac{1}{3} \mathbf{k} .
$$

If (23) is satisfied too, the special solutions correspond to the Third Harmonic Generation.

If $n^{\prime \prime \prime}=n^{\prime \prime}=n^{\prime}, \mathbf{k}_{*}^{\prime}=\mathbf{k}_{*}^{\prime \prime}=\mathbf{k}_{*}^{\prime \prime \prime}$ (and if (23) is satisfied), an analysis shows that $\zeta^{\prime}=\zeta^{\prime \prime}=\zeta^{\prime \prime \prime}$ is the only case that makes an essential difference compared with the case of a general function. If the rank of $\omega_{n^{\prime}}^{\prime \prime}\left(\mathbf{k}_{*}^{\prime}\right)$ is $d$, the rank of $\Phi^{\prime \prime}\left(\mathbf{k}_{*}^{\prime}, \mathbf{k}_{*}^{\prime}\right)$ is $2 d$. If the rank of $\omega_{n^{\prime}}^{\prime \prime}\left(\mathbf{k}_{*}^{\prime}\right)$ is $d-1$, then the rank of $\Phi^{\prime \prime}\left(\mathbf{k}_{*}^{\prime}, \mathbf{k}_{*}^{\prime}\right)$ in (45) is $2 d-2$. Therefore on the curve $\Xi$ at a generic point the Hessian is non-degenerate and the integral is of order $\varrho$. At several points where $\operatorname{det} \omega_{n^{\prime}}^{\prime \prime}\left(\mathbf{k}_{*}^{\prime}\right)=0$, that is on $\Xi^{\prime \prime}$, the integral is of order $\varrho^{5 / 3}$. Note that the manifolds $\Xi, \Xi^{\prime}$ and $\Xi^{\prime \prime}$ may be empty.

\subsubsection{Phase Function of Four-Wave Interactions With the Inversion (Spectral) Symmetry}

In this case for a given $(\bar{n}, \mathbf{k})$ the stronger interactions are attained for quads given by (30), that is $n^{\prime \prime \prime}=n^{\prime \prime}=n^{\prime}=n$, and (31), (32), or (33). The GVM conditions (26) and 
the FMC condition (23) are always satisfied. The manifold $\Xi(\vec{n})$ is obviously non-empty, and the index of an interacting quad is determined by the properties of the Hessian. The values of the indices are given below.

In the one-dimensional case, $d=1, \omega_{n}^{\prime \prime}(\mathbf{k})$ is a scalar. For almost all $\mathbf{k} \omega_{n}^{\prime \prime}(\mathbf{k}) \neq 0$, and, using (41), we get the integral of order $\varrho$. If $\omega_{n}^{\prime \prime}\left(\mathbf{k}_{*}\right)=0$, by (45) we get a point from $\Xi^{\prime \prime}$. Generically, the oscillatory integral is of order $\varrho^{2 / 3}, q_{0}=\frac{2}{3}$. Note that since FMC and GVM conditions are satisfied for any $\mathbf{k}$, we can find a point $\mathbf{k}_{*}$ such that $\omega_{n}^{\prime \prime}\left(\mathbf{k}_{*}\right)=0$ (even in the one dimensional case, $d=1$ ). Such $\mathbf{k}_{*}$ always exists since $\omega_{n}(\mathbf{k})$ is periodic and, hence, there always exists an inflection point. Therefore $\Xi^{\prime \prime}$ is non-empty and zerodimensional.

In the two-dimensional case, $d=2, d_{I}=4$ and $\omega_{n}^{\prime \prime}(\mathbf{k})$ is a symmetric $2 \times 2$ quadratic matrix with 3 independent coefficients. When the matrix $\omega_{n}^{\prime \prime}(\mathbf{k})$ has a zero eigenvalue, the second eigenvalue generically is not zero. The rank of $\Phi^{\prime \prime}\left(\mathbf{k}^{\prime}, \mathbf{k}^{\prime \prime}\right)$ in (45) in this case equals 2 , and we get a point of $\Xi^{\prime \prime}$. Note that the manifold $\Xi^{\prime \prime}$ is always non-empty. According to (31), (32), (33) the manifold $\Xi^{\prime \prime}$ consists of three curves in the $\left(\mathbf{k}, \mathbf{k}^{\prime}, \mathbf{k}^{\prime \prime}\right)$-plane, one of which is described by

$$
\operatorname{det} \omega_{n}^{\prime \prime}(\mathbf{k})=0, \mathbf{k}^{\prime}=\mathbf{k}^{\prime \prime}=\mathbf{k} .
$$

The function $\operatorname{det} \omega_{n}^{\prime \prime}(\mathbf{k})$ always has zeros thanks to the periodicity of $\omega_{n}(\mathbf{k})$. For modes from the curve $\Xi^{\prime \prime}$ we get the oscillatory integral of order $\varrho^{5 / 3}$, hence the interaction index $q_{0}=\frac{5}{3}$. This is the minimal interaction index possible in a generic case. Indeed, it is impossible to make $\omega_{1}^{\prime \prime}(\mathbf{k})=0$ in a generic case, since it requires vanishing of all three independent entries of the matrix $\omega^{\prime \prime}(\mathbf{k})$. One also cannot eliminate the cubic dependence in the two null directions by one parameter variation.

One more special case is described by Kerr type condition (35). In this case $\left(n^{\prime \prime} \neq n^{\prime}\right.$ or $\left.\mathbf{k}^{\prime \prime} \neq \mathbf{k}^{\prime}\right)$ generically $\Xi^{\prime \prime}$ is empty, and one may have a non-empty $(d-1)$-dimensional $\Xi^{\prime}, d=1,2$. Almost all points from $\Xi^{\prime}$ are of type $A_{2}$ with the index $q_{0}=d-\frac{1}{6}$ and in the case $d=2$ there may be several points of type $A_{3}$ with the index $q_{0}=d-\frac{1}{4}$.

\section{TABLES OF INTERACTION INDICES}

In this section we provide tables of interaction indices $q_{0}$ involved in (19), (20). The tables compare the oscillatory interaction integrals for the following types of the phase functions.

(i) General phase function. This phase function is an abstract, nonstructured function that is given for comparison purposes.

(ii) 4-frequency phase function of the form (17) with dispersion relations $\omega_{\bar{n}}(\mathbf{k})$ without the inversion (spectral) symmetry.

(iii) Phase function of the form (17) when dispersion relations $\omega_{\bar{n}}(\mathbf{k})$ have the inversion symmetry. 
Table 1. Indices of critical points for a generic one-dimensional periodic medium with a cubic nonlinearity.

\begin{tabular}{|c|c|c|c|c|c|}
\hline \multicolumn{3}{|c|}{ One-dimensional case $d=1$} \\
\hline $\begin{array}{c}\text { Phase function } \\
\text { type }\end{array}$ & General & $\begin{array}{c}\text { 4-frequency } \\
\text { without } \\
\text { inversion } \\
\text { symmetry }\end{array}$ & \multicolumn{4}{|c|}{$\begin{array}{c}\text { 4-frequency } \\
\text { with } \\
\text { inversion } \\
\text { symmetry }\end{array}$} \\
\hline Index $q_{0}$ & 1 & 1 & $\frac{2}{3}$ & $\frac{5}{6}$ & 1 \\
\hline $\begin{array}{c}\text { Interaction manifold } \\
\text { dimension }\end{array}$ & 0 & 0 & 0 & 0 & 1 \\
\hline
\end{tabular}

Table 2. Indices of critical points for a generic two-dimensional periodic medium with a cubic nonlinearity.

\begin{tabular}{|c|c|c|c|c|c|c|c|c|c|}
\hline & \multicolumn{9}{|c|}{ Two-dimensional case $d=2$} \\
\hline $\begin{array}{c}\text { Phase function } \\
\text { type }\end{array}$ & General & \multicolumn{2}{|c|}{$\begin{array}{c}\text { 4-frequency } \\
\text { without } \\
\text { inversion } \\
\text { symmetry }\end{array}$} & \multicolumn{5}{|c|}{$\begin{array}{c}\text { 4-frequences } \\
\text { with } \\
\text { inversion } \\
\text { symmetry }\end{array}$} \\
\hline Index $q_{0}$ & $\frac{11}{6}$ & 2 & $\frac{5}{3}$ & $\frac{11}{6}$ & 2 & $\frac{5}{3}$ & $\frac{7}{4}$ & $\frac{11}{6}$ & 2 \\
\hline $\begin{array}{c}\text { Interaction manifold } \\
\text { dimension }\end{array}$ & 0 & 1 & 0 & 0 & 1 & 1 & 0 & 1 & 2 \\
\hline
\end{tabular}

In all the three cases we assume that the phase function is generic, it does not have symmetries which are not explicitly imposed.

Every entry in the second row of the tables contains minimal values of $q_{0}$ for all possible interactions of strongly interacting quads. When several strongly interacting quads $(\bar{n}, \mathbf{k}),\left(\bar{n}^{\prime}, \mathbf{k}^{\prime}\right),\left(\bar{n}^{\prime \prime}, \mathbf{k}^{\prime \prime}\right),\left(\bar{n}^{\prime \prime \prime}, \mathbf{k}^{\prime \prime \prime}\right)$ with different $q_{0}$ occur for the same $(n, \mathbf{k})$, we choose only the minimal value of $q_{0}$ selecting the strongest interaction. Note that a smaller index $q_{0}$ corresponds to a higher intensity interaction. The third row in the tables gives the dimension of the interaction manifold in the $\mathbf{k}, \mathbf{k}^{\prime}, \mathbf{k}^{\prime \prime}$-space with the given value of $q_{0}$. Higher dimension means that more quads are involved in this type of interaction.

For example, let us look at the third column of the table describing the two-dimensional case. This column corresponds to media with the inversion symmetry and it has four subcolumns. The subcolumns correspond to 4 different values of $q_{0}=\frac{5}{3}, \frac{7}{4}, \frac{11}{6}, 2$. The largest $q_{0}=2$ since there is a 2-dimensional family $\Xi$ of quasimomenta $\mathbf{k}$ (that is almost all $\mathbf{k}$ ) with this index, consequently, the third row in this subcolumn has entry 2 . Then, there may exist a 1-dimensional family $\Xi^{\prime}$ (a curve) with $q_{0}=\frac{11}{6}$; there also may exist several points on $\Xi^{\prime}$ (a 0 -dimensional set) with $q_{0}=\frac{7}{4}$; and, finally, there always exists a spe- 
cial 1-dimensional family $\Xi^{\prime \prime}$ (a curve) of quasimomenta for which the interactions are the strongest, $\varrho^{q_{0}}=\varrho^{5 / 3}$ that is $q_{0}=5 / 3$.

\section{ACKNOWLEDGMENT AND DISCLAIMER}

The efforts of A. Babin and A. Figotin are sponsored by the Air Force Office of Scientific Research, Air Force Materials Command, USAF, under grant number F49620-011-0567. The US Government is authorized to reproduce and distribute reprints for governmental purposes notwithstanding any copyright notation thereon. The views and conclusions contained herein are those of the authors and should not be interpreted as necessarily representing the official policies or endorsements, either expressed or implied, of the Air Force Office of Scientific Research or the US Government.

\section{REFERENCES}

1. Optical gap solitons: Past, present, and future;theory and experiments, Chaos, 9(3), 584-589 (2000).

2. G. Agrawal, Nonlinear Fiber Optics (Academic Press, 1995).

3. N. Aközbek and S. John, Optical Solitary Waves in Two- and Three- Dimensional Nonlinear Photonic BandGap Structures, Phys. Rev. E, 57(2), 2287-2319 (1998).

4. J. Atai and B. A. Malomed, Bragg-grating solitons in a semilinear dual-core system, Physical Review E, 60(6), 8713-8718 (2000)

5. A. Arraf and C. M. de Sterke, Coupled-mode equations for quadratically nonlinear deep gratings, Physical Review E, 58(6), 7951-7958 (1998).

6. A. Arraf, H. He, and C. M. de Sterke, Deep Gratings with a $\chi^{(2)}$ Nonlinearity: Analysis and Solutions, Optical Fiber Technology, 5, 223-234 (1999).

7. A. Arraf, C. M. de Sterke, and H. He, Bright stationary solitary waves in deep gratings with a quadratic nonlinearity, Physical Review E, 63(2) (2001), 026611.

8. V. Belyakov, Diffraction Optics of Complex-Structured Periodic Medium (Springer-Verlag, 1992).

9. V. Berger, Nonlinear Photonic Crystals, Phys. Rev. Letters, 81, 4136-4139 (1998).

10. N. G. R. Broderick, G. W. Ross, H. L. Offerhous, D. J. Richardson, and D. C. Hanna, Hexagonally Poled Lithium Niobate: A Two-Dimensional Nonlinear Photonic Crystal, Physical Review Letters, 84(19), 4345-4348 (2000).

11. C. Conti, S. Trillo, and G. Assanto, Bloch function approach for parametric gap solitons, Optics Letters, 22(7), 445-447 (1997).

12. B. J. Eggleton, C. M. de Sterke, and R. E. Slusher, Nonlinear pulse propagation in Bragg gratings, J. Opt. Soc. Am. B, 14(11), 2980-2993 (1997).

13. Quasi-Phase-Matched Second Harmonic Generation: Tuning and Tolerances, IEEE J. of Quantum Electronics, 28(11), 2631-2654 (1992).

14. M. Goul'kov, S. Odoulov, I. Naumova, F. Agullo-Lopez, G. Calvo, E. Podivilov, B. Sturman, and V. Pruneri, Degenerate parametric light scattering in periodically poled LiNbO3 : Y : Fe, Physical Review Letters, 86(18), 4021-4024 (2001).

15. J. Haus, Viswanathan, M. Scalora., A. Kalocsai, J. Cole, and J. Theimer, Enhanced Second-Harmonic Generation in Media with a Weak Periodicity, Phys. Rev. A, 57(3), 2120-2128 (1998).

16. H. He and P. D. Drummond, Theory of multidimensional parametric band-gap simultons, Physical Review E, 58(4), 5025-5046 (1998).

17. T. Iizuka and C. M. de Sterke, Corrections to coupled mode theory for deep gratings, Physical Review E, 61(4), 4491-4499 (2000). 
18. J. Kumasako, M. Matsumoto, and S. Waiyapot, Linear stability analysis of dispersion-managed solitons controlled by filters, J. of Lightwave Technology, 18(8), 1064-1068 (2000).

19. D. Mills, Nonlinear Optics (Springer-Verlag, 1991).

20. S. Mou, C.-F. Lin, H.-F. Chou, Quasi-3-D beam -propagation method for modeling nonlinear wavelength conversion, J. of Lightwave Technology, 19(5), 772-779 (2001).

21. J. H. B. Nijhof, W. Forysiak, and N. J. Doran, The averaging method for finding exactly periodic dispersionmanaged solitons, IEEE J. of Selected Topics in Quantum Electronics, 6(2), 330-336 (2000).

22. M. Scalora, M. J. Bloemer, A. S. Manka, J. P. Dowling, C. M. Bowden, R. Viswanathan, and J. W. Haus, Pulsed second-harmonic generation in nonlinear, one-dimensional, periodic structures, Physical Review A, 56(4), 3166-3174 (1997).

23. M. J. Steel and C. M. de Sterke, Parametric amplification of short pulses in optical fiber Bragg gratings, Physical Review E, 54(4), 4271-4284 (1996).

24. C. M. de Sterke, D. G. Salinas, and J. E. Sipe, Coupled-mode theory for light propagation through deep nonlinear gratings, Physical Review E, 54(2), 1969-1989 (1996).

25. C. M. de Sterke, B. J. Eggleton, and P. A. Krug, High -intensity pulse propagation in uniform gratings and grating superstructures, J. of Lightwave Technology, 15(8), 1494-1502 (1997).

26. T. Yasui and M. Koshiba, Three-dimensional vector beam-propagation method for second harmonic generation analysis, J. of Lightwave Technology, 19(5), 780-785 (2001).

27. Y. Zhu and N. Ming, Dielectric Superlattices for Nonlinear Optical Effects, Optical and Quantum Electronics, 31, 1093-1128, (1999).

28. P. Butcher and D. Cotter, The Elements of Nonlinear Optics (Cambridge University Press, 1990).

29. A. Babin and A. Figotin, Nonlinear photonic crystals: I. Quadratic nonlinearity, Waves in Random Media, 2001.

30. N. Ashcroft and N. Mermin, Solid State Physics (Holt, Rinehart and Winston, New York-London, 1976).

31. M. Reed and B. Simon, Analysis of Operators, Vol. 4 (Academic Press, 1978).

32. W. Jones and N. M. March, Theoretical Solid State Physics Vols. 1,2 (Dover, New York, 1985).

33. N. Bleistein and R. A. Handelsman, Asymptotic Expansions of Integrals (Dover Publications, 1986).

34. V. I. Arnold, S. M. Gusein-Zade, and A. N. Varchenko, Singularities of Differentiable mappings, Vol. 1, Monographs in mathematics, 82 (Birkhauser, 1985).

35. V. I. Arnold, S. M. Gusein-Zade, and A. N. Varchenko, Singularities of Differentiable mappings, Vol. 2, Monographs in mathematics, 83 (Birkhauser, 1988). 